\title{
Preface
}

The growing number of individuals aged 65 and older will bring a relevant increase in the amount and costs of primary and specialty care for this group. The burden of diseases in this part of the population is mainly attributable to chronic conditions mainly related with risk factors connected with unhealthy lifestyles. Worldwide, hazardous and harmful alcohol use affects mostly middle aged individuals and older people, particularly men; the alcoholic beverages consumption represents the most common way of substance use by older adults. Many of the acute and chronic conditions that influence the high rates of health care use by older people are influenced by the consumption of alcohol; liver disease, cardiovascular disease, drugs or medication interactions, injury, memory problems, depression, cognitive changes and sleep problems may be considered a core selection of the long list of more than 60 diseases and cancers that have been showed to be fully or partially alcohol attributable. Due to the aging of the population and to the lifetime exposure to alcohol use, prevalence rates of substance use disorders are projected to increase dramatically. Over the past years, there has been a growing attention at European level on the relevance of alcohol use disorders in the elderly, as an increasing and common public health problem.

Alcohol consumption guidelines for the elderly are in progress in some European Member States, but apparently Italy is currently the only European country that has national nutritional recommendations for alcohol intake specifically addressed to older people that limit to 12 grams per day the daily alcohol use, whereas the other countries usually adopt the same recommendations used for adults (i.e. about $24 \mathrm{~g}$. of pure alcohol per day for males and 12 for females). Gender-and agespecific daily limits for alcohol consumption included in the Italian national guidelines for a healthy diet implemented by the Istituto Nazionale di Ricerca per gli Alimenti e la Nutrizione (Italian National Institute for Research on Foods and Nutrition) makes reference to already existing scientific evidences acknowledged by the Italian Ministry of Health, the Istituto Superiore di Sanità (ISS, Italian National Health Institute) and the Italian Society of Alcohology (SIA). The limits adopted in Italy for subjects aged 65 or more (no more than one standard drink, about 12 grams of pure alcohol, per day) are based on the consideration that ageassociated biological changes increases older people susceptibility to the negative effects of alcohol.
Although there is a paucity of epidemiological studies specifically aimed at identifying the impact of hazardous and harmful alcohol use in this vulnerable and growing part of the population, some estimates suggest that the prevalence of alcohol-related problems, alcohol abuse or dependence among older adults is increasing.

Some fully attributable alcohol diseases, such as alcoholic cirrhosis, as well as problems related to hazardous drinking are estimated to be even more common among older people than among younger individuals. With regard to alcohol use disorders, a huge gap between the number of older adults who should need treatment for addictive disorders and the number who are engaged in treatment is often reported by health care professionals, even if not subject to formal or scientific investigation.

A systematic screening for alcohol problems in the elderly is not a specific target of the ongoing prevention or action plans all over Europe, along with the early detection and brief intervention activities in primary health care, where professionals do not usually receive a clinical training focusing on the assessment of hazardous or harmful alcohol consumption among older people as an opportunity for prevention.

To what extent older people should be considered as a specific risk group in relation to alcohol is clearly an issue for debate and further research.

Some scientific findings have emphasized the relative higher impact of alcohol-related consequences and health outcomes among older people, such as changes in metabolism or in the pharmacokinetic processes that lead to an increasing vulnerability of ageing people to the ill effects of alcohol, the higher consumption of pharmaceutical drugs and the prevalence of diseases or health conditions in which alcohol use is not recommended; all arguments for stressing the special relationship between alcohol and older people. Most of the current scientific literature, even acknowledging the biological and metabolic differences between younger and older individuals, fails to elaborate data assuming a lower limit of at-risk consumption for older people, probably affecting the estimates of harmful drinking among older people. Further methodological problems in comparing studies aimed at evaluating the impact of harmful alcohol consumption are currently related to the age of reference, which varies widely from 55 to $65+$ or $80+$ years of age.

The VINTAGE project - Good Health into Older Age, funded by the European Commission under the 2nd Programme of Community Action in the Field of 
Health and coordinated by the ISS, originated from the assumption that despite the policy makers growing interest there is a substantial lack of comprehensive and harmonized data on the real impact of alcohol consumption on elderly subjects (evidence base), and also on cost-effective policies and programmes to prevent or reduce harmful alcohol consumption in this age class (experience base). In brief, the purpose of the VINTAGE project was to encourage evidence- and experience-based interventions to prevent the harmful use of alcohol amongst older people, by means of:

- systematic reviews of literature on alcohol and older people (369 titles identified, 78 relevant papers selected, results available in an online report);

- collection of examples of best practices to prevent harmful alcohol use among older people (ad hoc questionnaire sent to 309 EU experts, review of grey literature contained in 21 websites, results available online in 2 databases and a report);

- active dissemination of findings to those responsible for aging population or alcohol policy and programme development (project website, e-mail messages to a list serve of about 700 stakeholders, etc.).

The overall VINTAGE project experience identifies that more and better data and reporting, standardized across Europe, is needed on alcohol use, consumption patterns and alcohol-related consequences and health outcomes amongst older people.

The systematic review of scientific literature confirms the dearth of data and the need for further and more specific research. Further, although there is scarce evidence, it seems that older people respond equally well to alcohol policy measures, screening instruments and brief interventions as do younger adults. Given the importance of lifetime exposure, the maintenance of high frequency and high volume drinking of the middle age is likely to lead to upward pressure on the ill-health of the growing number of older people. For this reason, it should be recommended to overcome the distinction between current and future older people, and the target of alcohol policy should be also the middle aged.

The experience-based part of the VINTAGE study confirms that, despite a growing interest, alcohol use in the elderly is not yet perceived as a major issue for pre- vention in many realities in the EU. In fact, only a few of the reported initiatives were integrated in the system as permanent prevention strategies, or had been properly evaluated and published, and this might reduce the impact of the implemented efforts.

The policy implications arising from the VINTAGE project, whose results have been already submitted to debate at EU level, can be summarized as follows:

- alcohol and ageing is an issue for strategic framework of action and prevention;

- alcohol strategies should ensure an ageing perspective, as well as include alcohol issues into healthy ageing strategies;

- messages about alcohol consumption of older people (adults more in general) are politically sensitive;

- older people should be made more aware about alcohol-related consequences on their health and safety;

- alcohol consumption guidelines specific for elderly subjects should be an appropriate way of drawing attention to this apparently neglected target of health planning and prevention;

- alcohol in older people should be a major health policy issue to enhance healthy and active aging.

The increase in longevity is a tangible achievement of our times. On the other hand, it represents one of the greatest challenges for the immediate future. Only a systematic, urgent and adequate commitment, at policy, health and research level, will be able to turn this threat into an opportunity. The hope is that VINTAGE may contribute in raising awareness that a renewed approach should be ensured on alcohol and older people, in order to develop appropriate age-oriented interventions to be included both in the alcohol policy and the healthy and active aging policy agenda.

\section{Emanuele Scafato}

WHO Collaborating Centre for Research \& Health Promotion on Alcohol and Alcohol-Related Health Problems, Osservatorio Nazionale Alcol, Centro Nazionale di Epidemiologia, Sorveglianza e Promozione della Salute, Istituto Superiore di Sanità, Rome, Italy 